\title{
Processo de adaptação de aparelho de amplificação sonora individual: elaboração de um DVD para auxiliar a orientação a indivíduos idosos
}

\section{Adaptation process of hearing aids: preparation of a DVD to help assisting elderly individuals}

\author{
Karis de Campos ${ }^{1}$, Jerusa Roberta Massola de Oliveira ${ }^{2}$, Wanderléia Quinhoneiro Blasca ${ }^{3}$
}

\begin{abstract}
RESUMO
Objetivo: Estudar as dificuldades apresentadas pelos pacientes deficientes auditivos idosos relacionadas ao uso da amplificação, e elaborar um material didático em DVD, contendo informações sobre o uso e o manuseio do aparelho de amplificação sonora individual (AASI). Métodos: Participaram do estudo dez indivíduos idosos, com idade entre 65 a 80 anos, com deficiência auditiva do tipo neurossensorial de grau leve a moderado, novos usuários de AASI. Após o período de adaptação foi aplicado um questionário de múltipla escolha para avaliar as dificuldades encontradas. Resultados: Os resultados demonstraram que no processo de adaptação do AASI o individuo idoso apresenta maior dificuldade no uso e manuseio do aparelho e, principalmente, na inserção e remoção do molde auricular. A partir dos resultados, foi elaborado um material didático em DVD contendo informações importantes sobre o uso e manuseio do AASI. Conclusão: Analisando as dificuldades apresentadas pelos pacientes deficientes auditivos idosos é importante enfatizar a necessidade da elaboração de um material o didático sobre a orientação e a adaptação do aparelho objetivando o melhor aproveitamento da amplificação.
\end{abstract}

Descritores: Multimídia; Idoso; Auxiliares de audição; Perda auditiva bilateral; Reabilitação de deficientes auditivos

\section{INTRODUÇÃO}

O crescimento populacional vem se modificando continuamente. Hoje a característica da população mundial já não é mais a mesma encontrada há dez anos. No Brasil essa mudança tem se caracterizado pelo aumento do numero de idosos. Esse importante crescimento direciona modificações nos projetos de políticas públicas do país, evidenciando pesquisas para o envelhecer com melhor qualidade de vida ${ }^{(1)}$.

Trabalho realizado no Departamento de Fonoaudiologia da Faculdade de Odontologia de Bauru e na Divisão de Saúde Auditiva do Hospital de Reabilitação de Anomalias Craniofaciais, ambos da Universidade de São Paulo - USP - Bauru (SP), Brasil.

(1) Pós-graduanda (Mestrado) em Fonoaudiologia na Faculdade de Odontologia de Bauru da Universidade de São Paulo - USP - Bauru (SP), Brasil.

(2) Doutora, Fonoaudióloga da Divisão de Saúde Auditiva do Hospital de Reabilitação de Anomalias Craniofaciais da Universidade de São Paulo USP - Bauru (SP), Brasil.

(3) Doutora, Professora do Departamento de Fonoaudiologia da Faculdade de Odontologia de Bauru da Universidade de São Paulo - USP - Bauru (SP), Brasil.

Endereço para correspondência: Karis de Campos. R. Capitão Gomes Duarte, 20-40/402, Vl. Universitária, Bauru (SP), Brasil, CEP: 17012-226.

E-mail: karisdecampos@yahoo.com.br

Recebido em: 4/2/2009; Aceito em: 11/9/2009
O processo do envelhecimento é caracterizado por inúmeras alterações que refletem principalmente na comunicação do individuo, como a acuidade visual e auditiva, a destreza manual, além da repercussão psicossocial, devido à baixa auto estima ${ }^{(2)}$.

É importante ressaltar que existe uma grande prevalência de perda auditiva associada ao envelhecimento ${ }^{(3-6)}$. Quando há falha no mecanismo de interligação da ação fisiológica de ouvir às atividades periféricas e aos processos do sistema nervoso central ${ }^{(7)}$. A deficiência auditiva é o problema que produz maior impacto na comunicação ${ }^{(8-9)}$, propiciando 0 isolamento e hesitação nas situações sociais ${ }^{(10)}$. Somado a isso, a população idosa revela pior desempenho na comunicação, visto que a idade influencia no reconhecimento de fala ${ }^{(11-12)}$, necessitando, portanto, de maior atenção dos profissionais ${ }^{(13)}$.

Ao estabelecer um procedimento clínico de avaliação, de seleção e de adaptação do aparelho de amplificação sonora individual (AASI) para o idoso, o fonoaudiólogo deve ter, como principal objetivo, minimizar a desvantagem e/ ou a incapacidade auditiva ${ }^{(8)}$. A recomendação do AASI requer considerações referentes ao modelo, ao tipo de adaptação, à tecnologia e às características eletroacústicas ${ }^{(14)}$. Entretanto, a análise do desempenho não deve se restringir aos procedimentos clínicos. Informações sobre o uso efetivo nas 
diferentes situações de vida diária são também determinantes para o sucesso da adaptação.

Um estudo apontou que embora os idosos estejam preocupados com a perda auditiva, somente $8 \%$ da amostra optam por usar o AASI ${ }^{(15)}$. O deficiente auditivo, em geral, espera a deficiência agravar-se para procurar intervenção até não conseguir negar a deficiência auditiva ${ }^{(16)}$.

Pesquisadores da área de amplificação citam como motivos para a rejeição ao uso da amplificação: problemas financeiros, ruído excessivo, vaidade e, principalmente, dificuldade de manipulação do dispositivo ${ }^{(17-18)}$. Estes motivos indicam que fatores psicossociais devem ser considerados no processo de seleção e de adaptação do AASI reforçando a urgência de informação e de orientação objetivando o sucesso da adaptação.

Na reabilitação auditiva a motivação é fundamental para buscar ajuda e o auxílio para o uso adequado do AASI. A expectativa real em relação ao processo de adaptação é ponto fundamental em todo o trabalho. Assim, o papel do profissional é muito importante, orientando e direcionando para o melhor aproveitamento do dispositivo em beneficio da audição residual ${ }^{(8)}$.

As orientações direcionadas aos idosos exigem maior tempo em virtude da decadência sensorial e cognitiva ${ }^{(19)}$, portanto devem ser graduais, utilizando-se material como apoio para retenção e assimilação das informações. Se necessária, devem ser repetidas, e essa tarefa requer aptidão e boa vontade do profissional ${ }^{(13)}$. É importante enfatizar os aspectos relacionados à amplificação/molde auricular proporcionando independência ao usuário ${ }^{(20)}$. Este trabalho deve motivar o uso da amplificação.

Quando não houver orientação correta implicará na não utilização dos $\mathrm{AASIs}^{(13)}$. Desta forma, a criação e a implementação de programas de reabilitação ${ }^{(8)}$ podem ser uma alternativa.

Com o avanço tecnológico, surgem novas oportunidades para o desenvolvimento de ações educativas no processo de intervenção e reabilitação do paciente deficiente auditivo, buscando a melhora da qualidade de vida. Especificamente, em relação ao idoso, pesquisas realizadas têm recomendado a elaboração de material diversificado ${ }^{(21)}$, considerado como importante material de apoio.

Nesse contexto é importante considerar que o processo de aprendizagem sofre a influência de diversos aspectos e que há diferenças no modo como as pessoas aprendem. De acordo com essa visão, os aprendizes visuais preferem informações obtidas por meio de recursos ligados à representação por imagens: esquemas, gráficos e diagramas. Nos auditivos, a ênfase é dada pela informação ouvida. Observa-se que na prática, a maioria das pessoas usa uma combinação desses diferentes tipos de memória, mas referem preferência por um deles ${ }^{(22)}$.

$\mathrm{Na}$ área da saúde, durante o processo de orientação, muitos fatores acabam afetando a memória do indivíduo e prejudicando o processo de memorização. Estudos indicaram que, de toda a informação fornecida, apenas $50 \%$ é mantida; dessa porcentagem cerca de $40 \%$ a $80 \%$ podem ser esquecidas imediatamente. Com esses resultados, consideraram como uma estratégia importante o uso de materiais didáticos em multimídia para que o paciente possa levar para casa, com o objetivo de ser orientado novamente ${ }^{(23-24)}$.

Nos Estados Unidos, na Universidade de Minnesota, foi iniciado um projeto com o objetivo de elaborar materiais didáticos educacionais para pacientes e familiares ${ }^{(25)}$.

No Brasil, pesquisadores do grupo de Telessaude em Fonoaudiologia do Departamento de Fonoaudiologia da Faculdade de Odontologia de Bauru da Universidade de São Paulo foram os pioneiros no desenvolvimento de materiais didáticos para a orientação de usuário de AASI. Com uma experiência de mais de dez anos, sua atuação tem sido reconhecida nas comunidades cientificas nacionais e internacionais direcionando a uma nova proposta de atuação da fonoaudiologia. Portanto, o objetivo desse trabalho foi estudar as dificuldades dos deficientes auditivos idosos relacionadas ao uso da amplificação, e elaborar um material didático em multimídia (DVD), contendo informações sobre o uso e o manuseio do AASI.

\section{MÉTODOS}

Com o apoio do Programa de Educação Tutorial (PET) em Fonoaudiologia do Ministério da Educação, o estudo foi aprovado pelo Comitê de Ética em Pesquisa da Universidade de São Paulo, sob o processo de No 033/2007.

\section{Sujeitos}

Para o estudo, foram selecionados dez indivíduos idosos, cinco do sexo feminino e cinco do sexo masculino, com deficiência auditiva do tipo neurossensorial, grau leve/ moderado, bilateral e/ou unilateral, com idade entre 65 a 80 anos, novos usuários de aparelho de amplificação sonora individual do tipo retro-auricular (exceto adaptação aberta). Não foi levado em consideração o tipo e o material do molde auricular.

Para que os indivíduos pudessem fazer parte da pesquisa, teriam de ter a função cognitiva sem alterações; não apresentar nenhuma disfunção motora, como Doença de Parkinson ou sequela de Acidente Vascular Encefálico, por exemplo; ter acesso a um equipamento de DVD e saber utilizá-lo. Todos os pacientes que não se encaixavam em um desses requisitos não puderam participar do projeto.

\section{Procedimentos}

Com base em todos os tópicos referentes ao tema "Adaptação do aparelho de amplificação sonora individual", foi elaborado o questionário de múltipla escolha (Anexo 1). Esse questionário foi composto por 18 itens com duas possibilidades de escolha em cada um, em que cinco questões abordavam os aspectos gerais sobre o uso e o manuseio do aparelho e/ou molde auricular; 12 questões abordavam aspectos específicos das dificuldades apresentadas; e, para finalizar, uma questão direcionada à opinião do usuário.

Os indivíduos selecionados foram adaptados e receberam as orientações fonoaudiológicas sobre uso, cuidados e manuseio do AASI/molde auricular por uma fonoaudióloga e, 
após um mês de adaptação, retornaram para a revisão do AASI e para o acompanhamento, momento em que responderam ao questionário.

\section{Elaboração do material didático em DVD}

O conteúdo programático do DVD apresentado aos indivíduos que participaram da pesquisa foi elaborado a partir de uma completa revisão de literatura, abordando o tema "Aparelho de amplificação sonora individual".

Para a criação do material didático em DVD, foram necessários: a realização de um roteiro, sala adequada para a filmagem, filmadora (Sony®) e a maior gama possível de AASIs retro-auriculares e baterias, bem como molde auricular. Houve, também, a participação voluntária de duas pessoas: uma pessoa realizou a locução da teoria apresentada no material e a outra realizou a demonstração de como manusear e cuidar do AASI/molde auricular.

O conteúdo do DVD abordou informações específicas sobre a definição do AASI, sua importância, orientações sobre uso, inserção e remoção do dispositivo.

Para organização e montagem do filme, foi utilizado o programa Windows ${ }^{\circledR}$ Movie Maker versão 2003, no qual os vídeos foram editados pela autora e inseridos no mesmo programa, seguindo o roteiro previamente elaborado. Para facilitar o entendimento aos beneficiários, acrescentou-se legenda ao filme por meio do programa de legendas Adobe® Encore.

\section{RESULTADOS}

Os resultados serão apresentados em duas partes: avaliação das reais dificuldades do paciente deficiente auditivo idoso quanto ao uso adequado do AASI e a elaboração do material em DVD.

\section{Parte I - Avaliação das dificuldades apresentadas na adaptação do AASI}

No que se refere ao uso e ao manuseio do aparelho e do molde auricular, 50\% da amostra (cinco indivíduos) referiram apresentar esta dificuldade e $50 \%$ (cinco indivíduos) afirmaram que não a apresentavam. Este resultado também foi encontrado no item Molde auricular, como demonstrado na Figura 1. No que diz respeito à existência ou não de

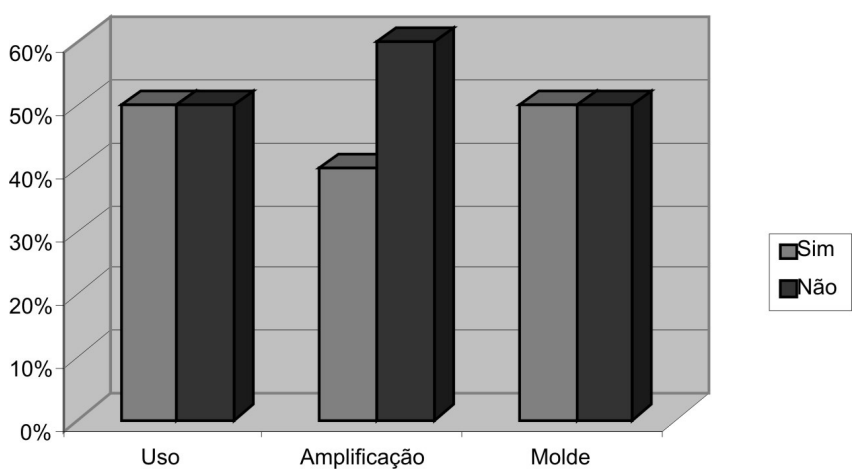

Figura 1. Dificuldade com o uso, amplificação e molde do AASI dificuldade com o AASI, especificamente, em relação à amplificação, 60\% (seis) dos indivíduos da amostra estudada responderam que não e $40 \%$ (quatro) dos indivíduos afirmaram ter tal dificuldade.

Em relação aos aspectos específicos relacionados à dificuldade quanto ao uso do AASI, foi possível verificar que, para realizar a conexão do gancho de som do aparelho e o molde auricular, $40 \%$ dos indivíduos apresentaram dificuldades em realizar a atividade, enquanto que $60 \%$ não apresentaram dificuldade. O mesmo resultado foi encontrado para o item da bateria, em que $40 \%$ dos indivíduos apresentaram dificuldade, enquanto que $60 \%$ dos indivíduos conseguiram realizar corretamente. Os indivíduos que fizeram parte da amostra foram indagados quanto às possíveis dificuldades do uso do AASI ao telefone. A esta questão responderam apenas sete indivíduos da amostra, pois os outros três não haviam testado o AASI ao telefone. Para o item da bobina telefônica, foi possível verificar que $42,86 \%$ (quatro) dos indivíduos apresentaram dificuldade. Quanto ao aspecto da bateria, 60\% (seis) dos indivíduos não apresentaram nenhuma dificuldade, enquanto $40 \%$ (quatro) apresentaram dificuldade, como demonstrado na Figura 2.

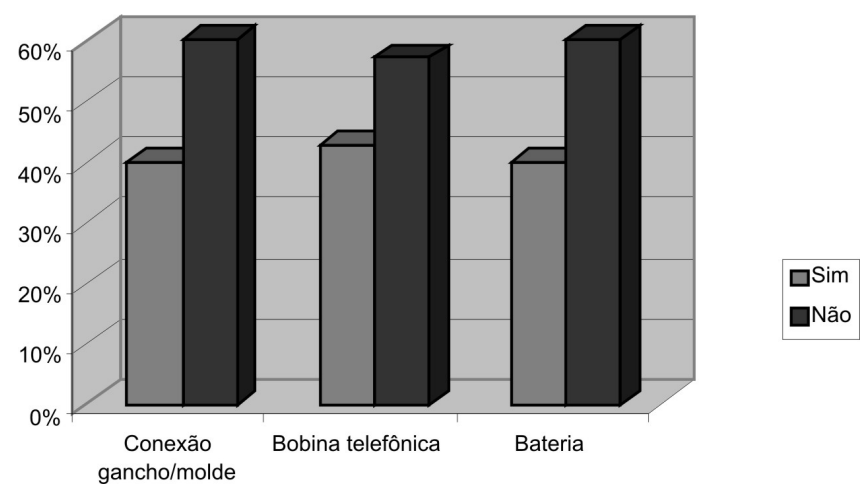

Figura 2. Dificuldades com relação ao manuseio do AASI por indivíduos idosos deficientes auditivos

Quanto ao aspecto relacionado ao molde auricular, $40 \%$ (quatro) dos indivíduos estudados mencionaram apresentar dificuldade em identificar orelha direita e orelha esquerda e $60 \%$ (seis) dos indivíduos relataram não apresentar esta dificuldade. O mesmo resultado foi verificado para o item de inserção e remoção do molde auricular. Já para o aspecto de higienização do molde auricular (lavar/secar), 70\% (7) dos indivíduos referiram não ter dificuldades em realizá-la e $30 \%$ (3) dos indivíduos relataram ter dificuldades, como demonstrado na Figura 3.

$\mathrm{Na}$ análise dos resultados apresentados na questão 13 , foi possível verificar que, dos dez indivíduos pesquisados, $70 \%$ (7) relataram necessitar da ajuda de alguém para manusear e/ ou cuidar do AASI e do molde auricular, enquanto que $30 \%$ (3) dos indivíduos demonstraram ter mais autonomia em relação ao AASI.

Quanto ao aspecto da importância da elaboração de um material didático para orientação, 80\% (8) dos indivíduos da amostra relataram sentir a necessidade de um material de apoio, enquanto que apenas $20 \%$ (2) dos indivíduos estudados não apresentaram esta necessidade. 


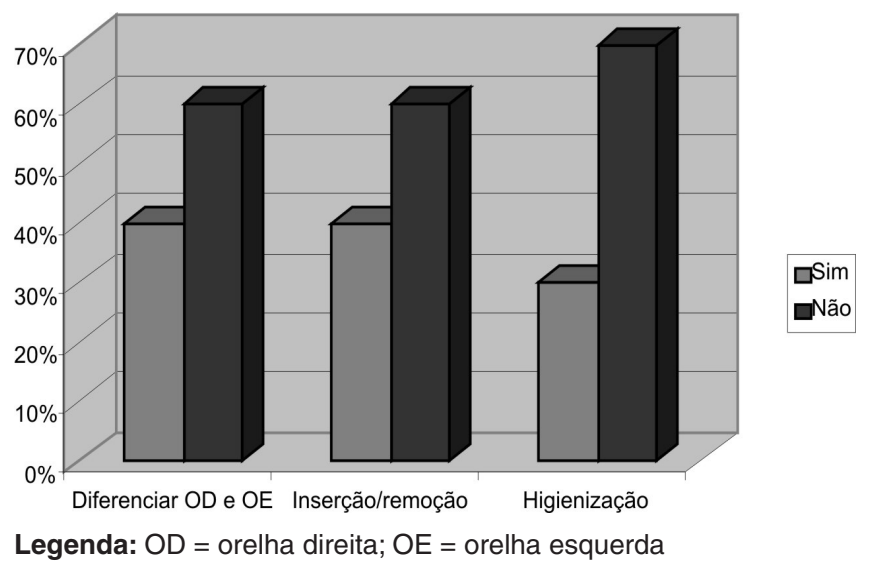

Figura 3. Dificuldades quanto ao molde auricular

\section{Parte II - Resultados da elaboração do material em multimídia}

O DVD elaborado abordou, de maneira abrangente, o tema adaptação do aparelho de amplificação sonora individual com o título "Conhecendo e Aprendendo sobre o seu Aparelho Auditivo".

Conteúdo abordado no DVD e sequência das cenas:

- Cena 1: O que é AASI?

- Cena 2: O molde auricular + limpeza

- Cena 3: Inserção e remoção do AASI

- Cena 4: Compartimento de bateria

- Cena 5: Chave liga/desliga e chave de seleção de entrada

- Cena 6: Controle de volume

- Cena 7: Controles internos para programação

Cena 8: Verificação e troca de bateria

Cena 9: Posição do microfone

Cena 10: Revisão dos procedimentos

- Cena 11: Posicionamento correto do molde no conduto auditivo externo

- Cena 12: Obstrução do conduto auditivo externo por cera Cena 13: Estojo

\section{DISCUSSÃO}

No processo de seleção e de adaptação do aparelho de amplificação sonora individual, o sucesso da adaptação está diretamente relacionado ao trabalho de orientação e de aconselhamento; no entanto, o profissional precisa conhecer as reais dificuldades dos pacientes em relação ao uso adequado da amplificação.

Assim, a presente pesquisa teve como objetivo estudar as dificuldades apresentadas pelos pacientes deficientes auditivos idosos, relacionadas ao uso adequado da amplificação e elaborar um material didático em multimídia (DVD), contendo informações sobre o uso e o manuseio do AASI.

Nessa pesquisa foi possível verificar que uma porcentagem expressiva dos indivíduos avaliados afirmou apresentar dificuldade quanto ao uso do aparelho. De acordo com os resultados apresentados na Figura 1, foi possível avaliar que, no que diz respeito à existência ou não de dificuldade com o AASI, especificamente, em relação aos aspectos avaliados, o uso e manuseio e molde auricular apresentaram maior porcentagem. Tais resultados corroboram estudos pregressos ${ }^{(12-13,19-20)}$, os quais apresentaram o manuseio do AASI como um dos itens que estão dentre as maiores dificuldades dos indivíduos idosos em relação à amplificação.

Durante todo o trabalho, faz-se necessário identificar as dificuldades específicas dos indivíduos, favorecendo, assim, o sucesso da amplificação. No projeto, os resultados obtidos demonstraram que as dificuldades em relação ao aspecto da conexão do gancho de som do aparelho, com o tubo do molde e a manipulação da bateria, foram os aspectos que apresentaram maior dificuldade, como demonstrado na Figura 2. Esses achados vão ao encontro dos trabalhos realizados ${ }^{(2)}$, que referem a alteração de destreza manual e tremores nas mãos como algumas das dificuldades do envelhecimento.

Como referido na literatura ${ }^{(26)}$, o uso do telefone colocouse como outro fator que dificulta a utilização do AASI, o que vem ao encontro do achado desta pesquisa, na qual $42,86 \%$ dos indagados mencionaram dificuldades no uso do dispositivo de bobina telefônica.

No que diz respeito às dificuldades específicas do molde auricular, as mais expressivas foram quanto à identificação de orelha direita e de orelha esquerda e à inserção e à remoção, obtendo-se uma porcentagem de $40 \%$. Os referidos resultados complementam um estudo que revela que alguns idosos desconheciam a função do molde auricular no processo de $\operatorname{adaptação~}^{(21)}$. Esses resultados podem estar relacionados à dificuldade de habilidade e de destreza manual, como também a alterações visuais do envelhecimento.

Quanto à necessidade de ajuda de outras pessoas para manusearem/cuidarem do AASI e molde auricular, $70 \%$ dos idosos relataram precisar desta ajuda. O mesmo dado foi encontrado em um estudo ${ }^{(26)}$, em que os indivíduos deficientes auditivos idosos precisaram da participação e do auxílio da família no processo de adaptação.

Na literatura, é possível encontrar trabalhos que enfatizam a importância dos aspectos emocionais no processo de seleção e de adaptação do AASI. O aspecto emocional é um fator de extrema importância, pois interfere na expectativa em relação aos benefícios da amplificação e no aprendizado sobre o uso do dispositivo (27).

Pensando na necessidade de transmitir conhecimentos específicos da adaptação do AASI, este trabalho teve por objetivo elaborar um material didático em multimídia, para que o mesmo pudesse, posteriormente, ser utilizado em um programa de Seleção e de Adaptação do AASI.

Desenvolver um material didático é uma tarefa desafiadora e desenvolver um material que proporcione o aprendizado, de forma agradável e interativa, é ainda mais complexo. Torna-se mais difícil e intrigante, quando pensamos no ser humano com todas as suas particularidades. E foi esta a razão que tornou este trabalho cada vez mais interessante.

A criação do DVD "Conhecendo e aprendendo sobre o meu aparelho auditivo" envolveu os alunos do Curso de Fonoaudiologia na elaboração de um conteúdo completo e de um roteiro direcionado ao aprendizado, o que favoreceu a construção de um material interativo e interessante, proporcionando ao paciente maior conhecimento sobre o tema abordado. 
Nos resultados encontrados na presente pesquisa, $80 \%$ da amostra julga importante e gostaria de adquirir um material didático em casa, sendo que ele deveria estar disponível para solucionar as dúvidas existentes durante todo o processo de adaptação. Tal fato revela a motivação maior por parte do indivíduo, que poderia ficar mais independente, conseguindo ter domínio sobre seu AASI. A maior habilidade com esse dispositivo refletir-se-á não só no aumento da adesão dessa população ao AASI, como também em maior facilidade clínica. Com um material didático disponível em casa, a responsabilidade da adaptação de aparelho de amplificação sonora individual será dividida com o usuário, tornando-o ativo nesse processo e não apenas restringindo-o às orientações do fonoaudiólogo em relação aos cuidados com o dispositivo.

Outro aspecto relevante é que, com o envelhecimento, o indivíduo necessita de maior tempo para a retenção de informações, precisando ouvir e visualizar várias vezes a mensagem para que ela seja armazenada, uma vez que pode haver presença de pequeno déficit de memória ${ }^{(19)}$. Devido a isto, faz-se importante a existência de um material didático, para que o usuário do AASI tenha acesso ao conteúdo quantas vezes ele julgar necessário ${ }^{(27-29)}$.

Sendo o aparelho de DVD um equipamento de baixo custo e, portanto, de fácil acesso, e sendo a sua utilização semelhante à de um aparelho de vídeo-cassete, é de grande valia realizar um material didático neste tipo de mídia. Para que o DVD fosse elaborado, houve, preliminarmente, a elaboração de um roteiro, que direcionou todos os aspectos a serem abordados da maneira mais didática para o paciente, que é leigo no assunto. Uma estruturação planejada do roteiro foi de grande importância ${ }^{(28-29)}$.

O DVD foi desenvolvido de modo que houvesse bastante apoio visual, com diversas demonstrações práticas, objetivando maior proximidade com a realidade. Os vídeos foram criados de forma que o indivíduo idoso conseguisse visualizar, de vários ângulos de imagem, os passos necessários para efetuar a manipulação e os cuidados adequados com o AASI. O dinamismo da apresentação tornou o conteúdo mais concreto e possível de ser imitado. Além disso, a presença de legenda foi imprescindível, utilizando a memória visual como forte aliada no processo de aprendizagem do conteúdo ${ }^{(30)}$.

Outro aspecto importante é o papel da memória auditiva no processo de retenção do conteúdo, em que a narração é de grande valia ${ }^{(22)}$. Esta observação do autor corrobora o cuidado atribuído ao desenvolvimento do material didático elaborado neste estudo, o qual contou com uma explanação narrativa de todos os tópicos abordados.

A linguagem utilizada no DVD foi simples e objetiva, de acordo com a linguagem dos indivíduos idosos usuários de AASI, que desconhecem o assunto. Tal aspecto possui alta relevância, uma vez que se faz necessário adequar a linguagem usada ao público alvo, para que ele compreenda a mensagem transmitida. Destaca-se, também, a importância da conversão da linguagem científica para a informal, com o intuito de atingir uma população leiga ${ }^{(30)}$.

Dentre as alterações gerais que o indivíduo idoso apresenta, há a alteração da destreza manual, principalmente no que se refere à coordenação motora fina. Pensando nisto, o DVD foi elaborado de forma que ele não precisasse apertar muitas teclas do controle remoto. O indivíduo precisará manusear apenas as teclas para colocar e tirar o DVD, já que todo o conteúdo é apresentado de uma única vez, em uma sequência lógica, e não em cenas.

Desta forma, a orientação à distância, com a aplicação de materiais didáticos como auxílio - a exemplo do DVD elaborado neste trabalho - deve ser pensada, para o indivíduo deficiente auditivo idoso, como uma estratégia adicional para o sucesso no processo de adaptação do AASI.

\section{CONCLUSÃO}

Uma parcela expressiva da amostra estudada possui dificuldades relacionadas com o manuseio e/ou cuidados com o AASI e molde auricular, enfatizando-se a necessidade da elaboração de um material didático sobre orientação e adaptação do AASI.

\begin{abstract}
Purpose: To study the difficulties faced by hearing impaired elderly patients related to the use of hearing aids, and to elaborate a didactic material on DVD, containing information about the use and handling of the hearing aid. Methods: Ten elderly subjects, with ages ranging from 65 to 80 years, new users of hearing aids with mild to moderate sensorineural hearing loss participated in the study. After the adjustment period, a multiple choice questionnaire was applied to assess the difficulties they faced. Results: Results showed that in the adaptation process with their hearing aids, elderly subjects have more difficulty to use and handle the apparatus and, mainly, to insert and remove the earmolds. Based on these results, a didactic material containing important information about using and handling hearing aids were elaborated on DVD. Conclusion: After considering the difficulties presented by hearing impaired elderly patients, it is important to emphasize the need for didactic material regarding orientation and adaptation of the hearing aids, in order to improve the use of amplification.
\end{abstract}

Keywords: Multimedia; Aged; Hearing aids; Hearing loss, bilateral; Rehabilitation of hearing impaired 


\section{REFERÊNCIAS}

1. Veras R. Em busca de uma assistência adequada à saúde do idoso: revisão da literatura e aplicação de um instrumento de detecção precoce e de previsibilidade de agravos. Cad Saúde Pública. 2003;19(3):705-15.

2. Quintero SM, Marotta RM, Marone SA. Avaliação do processamento auditivo de indivíduos idosos com e sem presbiacusia por meio do teste de reconhecimento de dissílabos em tarefa dicótica - ssw. Rev Bras Otorrinolaringol. 2002;68(1):28-33.

3. Gates GA, Cooper JC Jr, Kannel WB, Miller NJ. Hearing in the elderly: the Framingham cohort, 1983-1985. Part I. Basic audiometric test results. Ear Hear. 1990;11(4):247-56.

4. Parving A, Biering-Sørenson M, Bech B, Christensen B, Sørensen MS. Hearing in the elderly $>$ or $=80$ years of age. Prevalence of problems and sensitivity. Scand Audiol. 1997;26(2):99-106.

5. Uchida Y, Nakashima T, Ando F, Niino N, Shimokata H. Prevalence of self-perceived auditory problems and their relation to audiometric thresholds in a middle-aged to elderly population. Acta Otolaryngol. 2003;123(5):618-26.

6. Uimonen S, Maki-Torkko E, Jounio-Ervasti K, Sorri M. Hearing in 55 to 75 year old people in northern Finland-a comparison of two classifications of hearing impairment. Acta Otolaryngol Suppl.1997;529:69-70.

7. Luz SV, Pereira LD. Teste de escuta dicótica utilizando dígitos em indivíduos idosos. Acta AWHO. 2000;19(4):180-4.

8. Russo IC, Almeida K, Freire K. A reabilitação auditiva do idoso. In: Almeida K, Iorio MC. Próteses auditivas: fundamentos teóricos e aplicações clinicas. 2a ed. São Paulo: Lovise; 2003. p. 385-407.

9. Pinheiro MM, Pereira LD. Processamento auditivo em idosos: estudo da interação por meio de testes com estímulos verbais e não verbais. Rev Bras Otorrinolaringol. 2004;70(2):209-14.

10. Kerr PC, Cowie RI. Acquired deafness: a multi-dimensional experience. Br J Audiol. 1997;31(3):177-88.

11. Caporali SA, da Silva JA. Reconhecimento de fala no ruído em jovens e idosos com perda auditiva. Rev Bras Otorrinolaringol. 2004;70(4):52532.

12. Gordon-Salant S, Fitzgibbons PJ. Comparing recognition of distorted speech using an equivalent signal-to-noise ratio index. J Speech Hear Res. 1995;38(3):706-13.

13. Veras RP, Mattos LC. Audiologia do envelhecimento: revisão da literatura e perspectivas atuais. Rev Bras Otorrinol. 2007;73(1):128-34.

14. Russo IC, Almeida K. O processo de reabilitação audiológica do deficiente auditivo idoso. In: Marchesan IQ, Bollafi C, Gomes ID, Zorzi JL. Tópicos em fonoaudiologia. São Paulo: Lovise; 1994. p. 89-106.
15. Espmark AK, Rosenhall U, Erlandsson S, Steen B. The two faces of presbyacusis: hearing impairment and psychosocial consequences. Int $\mathrm{J}$ Audiol. 2002;41(2):125-35.

16. Oliveira VV, Blasca WQ. Avaliação do handcap em indivíduos idosos do centro dos distúrbios da audição, linguagem e visão do Hospital de Reabilitação de Anomalias Craniofaciais da USP. Salusvita 1999;18:7996.

17. Franks JR, Beckmann NJ. Rejection of hearing aids: attitudes of a geriatric sample. Ear Hear. 1985;6(3):161-6.

18. Russo IC. Intervenção fonoaudiológica na terceira idade. Rio de Janeiro:Revinter; 1998.

19. Rönnberg J. Cognition in the hearing impaired and deaf as a bridge between signal and dialogue: a framework and a model. Int J Audiol. 2003;42 Suppl 1:S68-76.

20. Oliveira JR, Motti TF, Neuber DR, Prado EP, Creppe SV, Andrade CF. Dificuldades encontradas pelos deficientes auditivos adultos, após a adaptação do aparelho de amplificação sonora individual (AASI). Acta AWHO. 2001;20(4):201-5.

21. Herrera LF, Blasca WQ. O molde auricular e suas implicações na adaptação do AASI no paciente deficiente auditivo idoso [monografia]. Bauru (SP): Instituto de Comunicação e Audição; 2005.

22. Hayden P. Learner's pocketbooks. $2^{\text {nd }}$ ed. Hampshire: Management Pocketbooks; 2005. p.46.

23. Margolis RH. What do your patients remember? Hear J. 2004;57(6):107.

24. Beck D, McGuire R. Advanced testing and fitting tools: techtopic. A multimedia: better tools facilitate a better process [internet] [cited 2009 Mar 20]. Available from: http://www.oticonusa.com/eprise/main/ SiteGen/Uploads/Public/Downloads_Oticon/The_Hearing_Review/ May_2006_HR.pdf

25. Audiology Incorporated [homepage on the internet] [cited 2009 Mar 8]. Available from: http://www.audiologyincorporated.com/ai_desc.htm

26. Barros PF, Queiroga BA. As dificuldades encontradas no processo de adaptação de aparelho de amplificação sonora individual em indivíduos idosos. Rev CEFAC. 2006;8(3):375-85.

27. Creppe SV, Blasca WQ. Análise da efetividade de um programa de adaptação do AASI para indivíduos deficientes auditivos idosos. J Bras Fonoaudiol. 2005;22(5):284-90.

28. Leleu-Merviel S, Labour M, Verclytte L, Vieville N. Script Creation for the Design of Lesson Plans. J Technol Teacher Educ. 2002;10(1):5-16.

29. Goodman RI. Evaluating scripts for instructional media programs: a structured script evaluation procedure. Educ Technol. 1984;24(3):25-30.

30. Miot HA, Paixão MP, Wen CL. Teledermatologia: passado, presente e futuro. An Bras Dermatol. 2005;80(5):523-32.

Anexo 1. Protocolo de avaliação

Data:

Nome:

Modelo AASI:

Modelo molde:

( ) acrílico

( ) silicone

Atendimento:

Parte 1

1 - Quanto tempo faz que você usa o aparelho auditivo?

() 1 mês () 3 meses () mais de 6 meses () 1 ano () mais de 1 ano

2 - Quantas horas por dia você usa o aparelho auditivo?

( ) 1 hora ( ) 2 horas ( ) 4 horas ( ) 8 horas ( ) o dia todo

3 - Você tem dificuldade em manusear o aparelho?

( ) $\operatorname{Sim}$

( ) Não
4 - Você tem dificuldade com relação a amplificação do aparelho?

( ) $\operatorname{Sim}$ ( ) Não

5 - Você apresenta dificuldade relacionada ao molde auricular?

( ) Sim ( ) Não

Parte 2

1 - Apresenta dificuldade em identificar orelha direita e orelha esquerda?

( ) Sim ( ) Não 
2 - Você tem dificuldade em colocar o molde no gancho do aparelho auditivo?
( ) $\mathrm{Sim}$
( ) Não

3 - Você tem dificuldade em retirar o molde do gancho do aparelho auditivo?

( ) Sim

( ) Não

4 - Tem alguma dificuldade em colocar o aparelho auditivo na orelha?

( ) $\mathrm{Sim}$

( ) Não

5 -Tem alguma dificuldade em retirar o aparelho auditivo da orelha?
( ) Sim
( ) Não

6 - Tem dificuldade em usar a bobina telefônica?

( ) Sim ( ) Não

7 - Tem dificuldade de lavar o molde auricular?

$\begin{array}{ll}\text { ( ) } \operatorname{Sim} & \text { ( ) Não }\end{array}$

8 - Tem dificuldade de secar o molde auricular?
( ) $\operatorname{Sim}$
( ) Não

9 - Consegue fechar o lugar onde fica a pilha?

( ) Sim ( ) Não

10 - Consegue abrir o lugar onde fica a pilha?

( ) $\operatorname{Sim}$ ( ) Não

11 - Consegue trocar a pilha corretamente?

( ) Sim ( ) Não

12 - Precisou da ajuda de alguém para mexer no aparelho auditivo?

( ) Sim ( ) Não

Parte 3

1 - Você acha importante ter um material para levar para casa, que pudesse esclarecer suas dúvidas quanto ao uso e manuseio do AASI?

( ) Sim

( ) Não 